CrossMark

$<$ click for updates

Cite this: Food Funct., 2016, 7, 3141

\title{
Polymer-free nanofibers from vanillin/cyclodextrin inclusion complexes: high thermal stability, enhanced solubility and antioxidant property $\dagger$
}

\author{
Asli Celebioglu, a,b Fatma Kayaci-Senirmak, ${ }^{\text {a,b }}$ Semran İpek, ${ }^{b, c}$ Engin Durgun ${ }^{a, b}$ and \\ Tamer Uyar*a,b
}

Vanillin/cyclodextrin inclusion complex nanofibers (vanillin/CD-IC NFs) were successfully obtained from three modified CD types (HP $\beta C D, H P \gamma C D$ and $M \beta C D$ ) in three different solvent systems (water, DMF and DMAc) via an electrospinning technique without using a carrier polymeric matrix. Vanillin/CD-IC NFs with uniform and bead-free fiber morphology were successfully produced and their free-standing nanofibrous webs were obtained. The polymer-free CD/vanillin-IC-NFs allow us to accomplish a much higher vanillin loading $(\sim 12 \%, w / w)$ when compared to electrospun polymeric nanofibers containing CD/vanillin-IC $(\sim 5 \%, w / w)$. Vanillin has a volatile nature yet, after electrospinning, a significant amount of vanillin was preserved due to complex formation depending on the $C D$ types. Maximum preservation of vanillin was observed for vanillin/M $\beta C D-I C$ NFs which is up to $~ 85 \% \mathrm{w} / \mathrm{w}$, besides, a considerable amount of vanillin

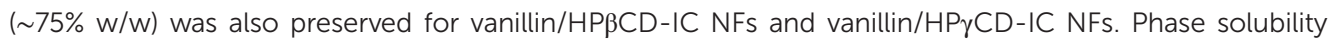
studies suggested a 1:1 molar complexation tendency between guest vanillin and host CD molecules. Molecular modelling studies and experimental findings revealed that vanillin: $C D$ complexation was

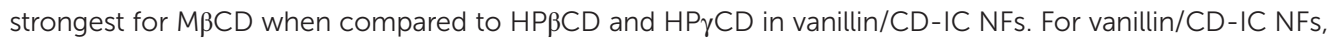
water solubility and the antioxidant property of vanillin was improved significantly owing to inclusion complexation. In brief, polymer-free vanillin/CD-IC NFs are capable of incorporating a much higher loading of vanillin and effectively preserve volatile vanillin. Hence, encapsulation of volatile active agents such as flavor, fragrance and essential oils in electrospun polymer-free CD-IC NFs may have potential for food related applications by integrating the particularly large surface area of NFs with the non-toxic nature of $C D$ and inclusion complexation benefits, such as high temperature stability, improved water solubility and an enhanced antioxidant property, etc.

Received 21st April 2016 Accepted 14th June 2016 DOI: $10.1039 /$ c6fo000569a www.rsc.org/foodfunction antimutagenic and antioxidant properties. $^{3-5}$ However, thermal instability and volatility of vanillin is a serious difficulty during its use and processing. In order to overcome this problem, one option is the molecular encapsulation of vanillin into cyclodextrin by inclusion complexation which increases the stability of vanillin at higher temperatures for a longer shelf life. ${ }^{5-8}$

Cyclodextrins (CDs) are one of the most intriguing oligosaccharide types due to their cyclic and truncated cone shape structures. These molecules gain their unique features by the enzymatic degradation process of starch and three of them come into prominence, $\alpha-\mathrm{CD}, \beta-\mathrm{CD}$ and $\gamma$-CD, which are composed of 6,7 , and 8 glucopyranose subunits, respectively. ${ }^{9,10}$ Apart from these three native $\mathrm{CD}$ types, different $\mathrm{CD}$ derivatives are also obtained by chemical or enzymatic modifications allowing for higher solubility, different cavity volume and stability against light and oxygen. ${ }^{11}$ Hydroxypropyl- $\beta$-cyclodextrin (HP $\beta \mathrm{CD})$, hydroxypropyl- $\gamma$-cyclodextrin (HP $\gamma \mathrm{CD}$ ) and 
methyl- $\beta$-cyclodextrin (M $\beta \mathrm{CD}$ ) are the most common modified $\mathrm{CD}$ types due to their scalability for industrial production, standardization and availability. ${ }^{10,11}$

Owing to its intriguing molecular structure, CD offers a relatively hydrophobic inner cavity and hydrophilic outer surface that enables it to form noncovalent host guest interactions (inclusion complexes (ICs)) with a variety of molecules. Food additives, such as flavors/fragrances, are one of the potential guest molecules ${ }^{10}$ that can be encapsulated by a CD cavity, which ensures stabilization and protection from evaporation, degradation and oxidation in addition to controlling and/or delaying release. ${ }^{10-12}$ The size and shape of the CD cavity are highly influential for complexation, so different types of $\mathrm{CD}(\alpha-\mathrm{CD}, \beta-\mathrm{CD}$ and $\gamma-\mathrm{CD})$ would have different stabilities with the same guest molecules. ${ }^{13}$ There are studies in the literature regarding to complexation between vanillin and different kinds of CD types. ${ }^{5,8,14-16}$ Even in one of the related study, we have reported the detailed investigation of solid state vanillin/CD-IC by using native $\mathrm{CD}(\alpha$-CD, $\beta$-CD and $\gamma$-CD) and in this report, the enhancement of thermal stability and sustained release of vanillin have been observed. ${ }^{6}$

The strategy of incorporation of CD-IC into a high surface area nanofiber matrix may offer practical applications. Electrospinning is already the center of interest for many application areas by making possible the production of nanofibers (NFs) having very high surface areas with unique and variable physicochemical properties. ${ }^{17,18}$ This technique also enables high encapsulation/storage efficiency for a functional compound that leads to a significant amount of food and active food packaging applications. From this point of view, a few studies have been reported in the literature about the incorporation of food additive/CD-IC with electrospun NFs and significant studies were performed by our group. ${ }^{19-25}$ For instance, we have produced functional polyvinyl alcohol (PVA) electrospun NF incorporating vanillin/CD-IC by using three types of $\mathrm{CD}(\alpha-\mathrm{CD}, \beta-\mathrm{CD}$ and $\gamma$-CD) and high thermal stability, enhanced durability as well as a carrier template were provided for vanillin and its IC. ${ }^{19}$ In other related studies, CD-IC of eugenol, ${ }^{20}$ geraniol $^{21}$ and gallic acid ${ }^{25}$ which are wellknown food additives, were also incorporated into polymeric NFs to integrate the advantages of both $\mathrm{CD}$ and electrospun NFs.

However, in the case of the polymeric matrix used, the loading of the active agents in NF is limited between 3 to $5 \%$ (w/w, with respect to the fiber matrix) since incorporation of a higher amount of CD-IC $(50 \% \mathrm{w} / \mathrm{w}$, which corresponds to 3 to $5 \%$ of active agent) often creates a serious problem for the electrospinning of uniform nanofibers. Additionally, the polymer types used could also be a concern related to the practices for food applications. So, electrospinning of active agents of CD-IC nanofibers without using a polymer matrix would be quite advantageous due to the non-toxic nature of $\mathrm{CD}$ with the ability to form an inclusion complex which provides high temperature stability, improved water solubility and an enhanced antioxidant or antibacterial property. Moreover, much higher loading of active agents such as flavor, fragrance and essential oils can be efficiently encapsulated in electrospun polymer-free CD-IC NFs and these active agents can be effectively preserved for a longer time due to inclusion complexation.

Recently, we have reported the possibility of electrospinning of uniform nanofibers and their free-standing nanowebs from $\mathrm{CD}$ molecules (both native $(\alpha-\mathrm{CD}, \beta-\mathrm{CD}, \gamma-\mathrm{CD}))^{26,27}$ and modified CD (HP $\beta C D, H P \gamma C D$ and $\mathrm{M} \beta \mathrm{CD})^{28-30}$ without using a carrier polymeric matrix. Moreover, we were successful at electrospinning of polymer-free nanofibrous webs from cyclodextrin inclusion complexes with triclosan (antibacterial agent). ${ }^{31,32}$ For the electrospinning of $\mathrm{CD}$ by itself without using a carrier polymeric matrix, we find out that the primary criterion is the self-assembly and self-aggregation of these supramolecular CD molecules in their highly concentrated solutions.

Here, electrospinning of polymer-free vanillin/CD-IC NFs was carried out by using three CD types (HP $\beta C D, H P \gamma C D$ and $\mathrm{M} \beta \mathrm{CD}$ ). As such, we attained appropriate materials for food applications along with the non-toxic and edible nature of $\mathrm{CD}$. The inclusion complexation between $\mathrm{CD}$ and vanillin was prepared in three different solvents: water, DMF and DMAc. The morphology characterization of free-standing nanowebs was done by scanning electron microscopy (SEM). The detailed characterization of vanillin/CD-IC NFs was carried out by using Fourier transform infrared spectrometry (FTIR), X-ray diffraction (XRD), differential scanning calorimetry (DSC) and thermogravimetric analysis (TGA). The molar ratios between vanillin and $\mathrm{CD}(\mathrm{HP} \beta \mathrm{CD}, \mathrm{HP} \gamma \mathrm{CD}$ and $\mathrm{M} \beta \mathrm{CD}$ ) were calculated by using the proton nuclear magnetic resonance $\left({ }^{1} \mathrm{H}-\mathrm{NMR}\right)$ technique. The ab initio computational modeling studies were performed for inclusion complexation of vanillin and $\mathrm{CD}$ in an aqueous system. In addition to phase solubility studies, water solubility and the antioxidant property of vanillin/CD-IC NF web samples were investigated.

\section{Experimental}

\subsection{Materials}

Vanillin (99\% purity, Sigma-Aldrich) was obtained commercially. Hydroxypropyl- $\beta$-cyclodextrin (HP $\beta$ CD) (degree of substitution: $\sim 0.6$, Cavasol@W7 HP Pharma), hydroxypropyl$\gamma$-cyclodextrin (HP $\gamma \mathrm{CD}$ ) (degree of substitution: $\sim 0.6$, Cavasol@W8 HP) and methyl- $\beta$-cyclodextrin (M $\beta \mathrm{CD}$ ) (degree of substitution: 1.6-1.9 Cavasol®W7 M Pharma) were kindly donated by Wacker Chemie AG (Germany). Potassium bromide (KBr, 99\%, FTIR grade, Sigma-Aldrich), deuterated dimethylsulfoxide (d6-DMSO, deuteration degree min. 99.8\% for NMR spectroscopy, Merck), dimethylacetamide (DMAc) (SigmaAldrich, 99\%) and N,N-dimethylformamide (DMF) (Rie-del, Pestenal), methanol (extra pure, Sigma Aldrich), 2,2-diphenyl1-picrylhydrazyl (DPPH, Sigma Aldrich) were used in this study. The water used was from a Millipore Milli-Q ultrapure water system. The materials were used as-received without any further purification process. 


\subsection{Preparation of electrospinning solutions}

The inclusion complexes (ICs) of vanillin with $\mathrm{HP} \beta \mathrm{CD}, \mathrm{HP} \gamma \mathrm{CD}$ and $\mathrm{M} \beta \mathrm{CD}$ were aimed to be prepared by using a 1:1 molar ratio of vanillin : $\mathrm{CD}$ in water, DMF and DMAc. The optimal $\mathrm{CD}$ concentrations for the electrospinning of HP $\beta \mathrm{CD}, \mathrm{HP} \gamma \mathrm{CD}$ and $\mathrm{M} \beta \mathrm{CD}$ in water, DMF and DMAc were determined in our previous study. ${ }^{29}$ First of all, vanillin powder was put in water, DMF and DMAc; vanillin was dissolved immediately in DMF and DMAc, however it was only dispersed in water (Fig. 1a). Then HP $\beta C D(160 \%(w / v)$ in water), $120 \%(w / v)$ in $\mathrm{DMF}$ and $120 \%(\mathrm{w} / \mathrm{v})$ in DMAc, HP $\gamma$ CD $(160 \%(\mathrm{w} / \mathrm{v})$ in water), $125 \%(\mathrm{w} / \mathrm{v})$ in DMF and $125 \%(\mathrm{w} / \mathrm{v})$ in DMAc and M $\mathrm{DCD}(160 \%(\mathrm{w} / \mathrm{v})$ in water), $160 \%(\mathrm{w} / \mathrm{v})$ in DMF and $160 \%(\mathrm{w} / \mathrm{v})$ in DMAc were separately added to the vanillin systems and they were stirred overnight and water based solutions also became clear and homogeneous due to the dissolution of vanillin by inclusion complex formation (Fig. 1a).

\subsection{Electrospinning of nanofibers (NFs)}

Each vanillin/CD-IC solution was placed in a plastic syringe ( 1 or $3 \mathrm{~mL}$ ) fitted with a metallic needle $(0.4$ or $0.6 \mathrm{~mm}$ inner diameter). The syringe was fixed horizontally on the syringe pump (KD Scientific, KDS 101). The electrode of the high-

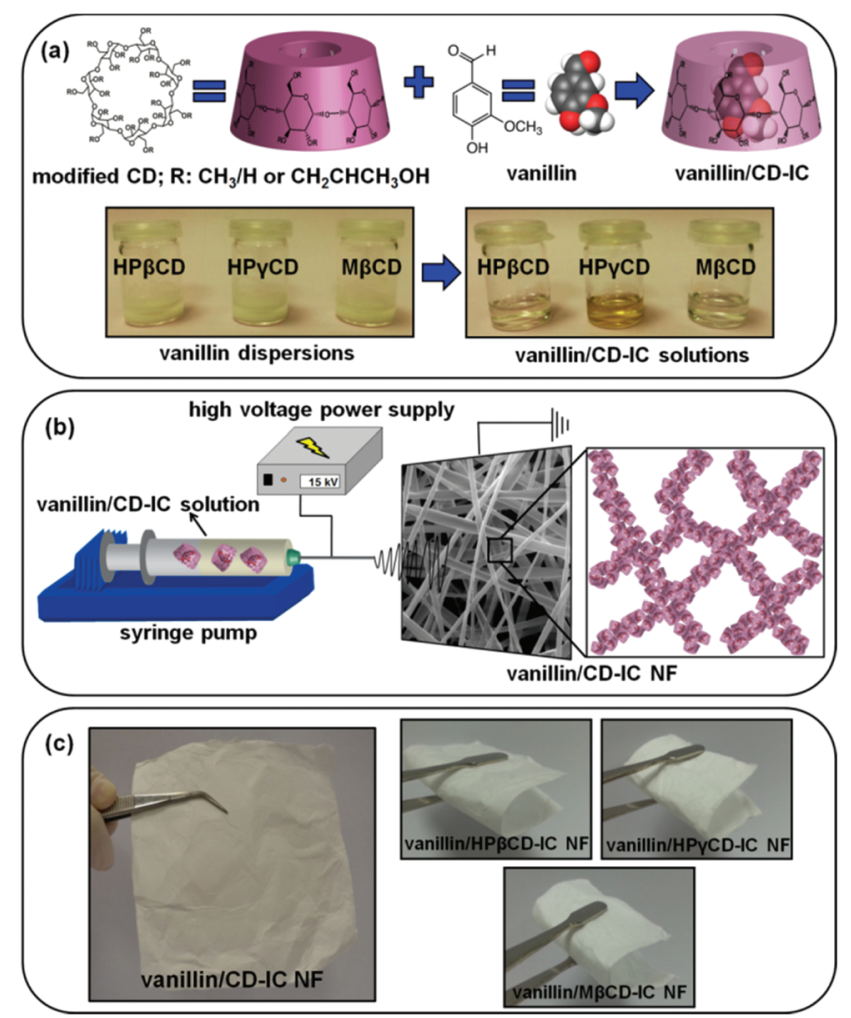

Fig. 1 (a) Chemical structure of modified CD and vanillin molecules, schematic representation of IC formation between CD and vanillin, and photographs of water based vanillin dispersion and vanillin/CD-IC solutions. (b) Schematic representation of the electrospinning of vanillin/CD-IC NFs. (c) Photographs of electrospun vanillin/CD-IC nanofibrous webs. voltage power supply (Spellman, SL Series) was clamped to the metal needle tip, and the plate type aluminum collector was grounded. The feed rate of solutions was between 0.5-1 $\mathrm{mL} \mathrm{h}^{-1}$, the applied voltage was between 10-15 kV, and the tip-to-collector distance was kept between 10-20 cm. Electrospun NFs were deposited on a grounded stationary metal collector covered with a piece of aluminum foil. The electrospinning apparatus was enclosed in a Plexiglas box, and electrospinning was carried out at $25{ }^{\circ} \mathrm{C}$ at $30 \%$ relative humidity.

\subsection{Measurements and characterization}

A rheometer (Anton Paar Physica MCR 301) equipped with a cone/plate accessory (spindle type CP40-2) was used to measure the rheological behavior of the vanillin/CD-IC solutions in the range of 0.1 to $100 \mathrm{~Pa}$ shear stress at $22{ }^{\circ} \mathrm{C}$. Scanning electron microscopy (SEM, FEI-Quanta 200 FEG) was used for the morphological investigation of the electrospun NFs. Samples were sputtered with $5 \mathrm{~nm} \mathrm{Au} / \mathrm{Pd}$ prior to SEM imaging. The average fiber diameter (AFD) was determined from the SEM images, and around 100 fibers were analyzed. The infrared spectra of the samples were obtained using Fourier transform infrared spectrometry (FTIR, BrukerVERTEX70). The samples were mixed with potassium bromide $(\mathrm{KBr})$ and pressed as pellets. The scans (64 scans) were recorded between 1800 and $900 \mathrm{~cm}^{-1}$ at a resolution of $4 \mathrm{~cm}^{-1}$. X-ray diffraction (XRD) (PANalytical X'Pert powder diffractometer) data of the vanillin and vanillin/CD-IC NF were recorded by using $\mathrm{Cu} \mathrm{K} \alpha$ radiation in a range of $2 \theta=5^{\circ}-30^{\circ}$. Differential scanning calorimetry (DSC, TA Q2000) and thermogravimetric analysis (TGA, TA Q500) were used for the investigation of the thermal properties of the samples. DSC analyses were carried out under nitrogen. Initially, samples were equilibrated at $0{ }^{\circ} \mathrm{C}$ and then heated to $200{ }^{\circ} \mathrm{C}$ at a heating rate of $10{ }^{\circ} \mathrm{C} \mathrm{min}^{-1}$. TGA of the samples was carried out from 25 to $500{ }^{\circ} \mathrm{C}$ at a $20^{\circ} \mathrm{C} \mathrm{min}^{-1}$ heating rate, and nitrogen was used as a purge gas. The molar ratio between vanillin : CD was determined by using proton nuclear magnetic resonance $\left({ }^{1} \mathrm{H}-\mathrm{NMR}\right.$, Bruker D PX-400) system. The electrospun vanillin/CD-IC NFs were dissolved in d6-DMSO at a $20 \mathrm{~g} \mathrm{~L}^{-1}$ concentration. The spectra were recorded at $400 \mathrm{MHz}$ and for 16 total scans. Integration of the chemical shifts $(\delta)$ given in parts per million ( $\mathrm{ppm}$ ) of the samples was calculated by using Mestrenova software.

Phase solubility analyses were carried in aqueous medium according to the method of Higuchi and Connors. ${ }^{33} 10 \mathrm{~mL}$ of aqueous CD solutions were prepared ranging from 0 to $60 \mathrm{mM}$ concentration and an excess amount of vanillin was added to each of these systems. The solutions were stirred for $24 \mathrm{~h}$ at RT to reach equilibrium. Then, all suspensions were filtered through a $0.45 \mu \mathrm{m}$ membrane filter to remove the undissolved solid part of vanillin. Vanillin concentration with respect to increasing CD concentration was determined by using UV-Vis spectroscopy (Varian, Carry 100) at $278 \mathrm{~nm}$. The phase solubility diagrams are plots of the molar concentration of vanillin versus molar concentration of $\mathrm{CD}$. 
The water solubility limit of vanillin is known as $10 \mathrm{mg} \mathrm{mL}^{-1} \cdot{ }^{16}$ Here, we have tried to prepare a pure vanillin solution at $20 \mathrm{mg} \mathrm{mL} \mathrm{m}^{-1}$ concentration. At the same time, we have dissolved vanillin/CD-IC NFs in water produced from three CD types (HP $\beta C D$, HP $\gamma \mathrm{CD}$ and $\mathrm{M} \beta \mathrm{CD}$ ) having the same concentration of vanillin $\left(20 \mathrm{mg} \mathrm{mL}^{-1}\right)$. We have observed that, while vanillin/CD-IC NFs were dissolved immediately, there was still an insoluble part of vanillin in the pure solution even after stirring overnight. The solubility determination of the samples was performed by using high performance liquid chromatography (HPLC, Agilient, 1200 series). Before the HPLC measurement, all vanillin/CD-IC solutions and vanillin dispersion were filtered to remove the undissolved parts of vanillin. The filtered solutions were loaded onto the HPLC system and the separation of vanillin was performed with a Zorbax Eclipse XDB-C18 column $(150 \mathrm{~mm} \times 4.6 \mathrm{~mm}, 5 \mu \mathrm{m}$ particle size) and it was detected at a $254 \mathrm{~nm}$ wavelength. Water: methanol $(40: 60)$ was used as the mobile phase at a flow rate of $0.3 \mathrm{~mL} \mathrm{~min}^{-1}$ and the injection volume was kept at $1 \mu \mathrm{l}$. The calibration curve of vanillin was prepared from stock solutions at 10 different concentrations from $10 \mathrm{mg} \mathrm{mL} \mathrm{m}^{-1}$ to $20 \mu \mathrm{g} \mathrm{mL}^{-1}$. It showed linearity and acceptability with $R^{2} \geq 0.99$. The experiments were performed in triplicate.

Antioxidant activity of the samples was examined by the 2,2-diphenyl-1-picrylhydrazyl (DPPH) radical scavenging method. For this, a DPPH ${ }^{*}$ solution in methanol $(1.2 \mathrm{mM})$ was freshly prepared and $2.3 \mathrm{~mL}$ of this solution was mixed with $200 \mu \mathrm{L}$ of aqueous solutions of water based vanillin/CD-IC NFs and pure vanillin. While the ultimate solution concentration of vanillin/CD-IC NF was adjusted to $12 \mathrm{mg} \mathrm{mL} \mathrm{m}^{-1}$, pure vanillin concentration was determined from the highest initial vanillin content amount $(12 \%, \mathrm{w} / \mathrm{w}$ with respect to the total sample amount) which was used for the vanillin/M $\beta C D-I C$ system. The vanillin/CD-IC NFs were dissolved immediately in water, however in case of the pure vanillin system, we had to filter the solution to eliminate the undissolved vanillin part. The $\mathrm{DPPH}^{*}$ solution has maximum absorption at $517 \mathrm{~nm}$ and, along with a reduction by the antioxidant compound, this dominant absorbance disappears. Thus, after the addition of the sample solution into the DPPH stock solution, a reduction at the absorbance intensity $(517 \mathrm{~nm})$ was recorded at progressing time intervals by using UV-Vis spectroscopy (Varian, Carry 100). The experiment was carried out in triplicate and the DPPH radical scavenging efficiency was expressed as the inhibition percentage and was calculated by the following formula:

$$
\begin{aligned}
\mathrm{DPPH} \text { radical scavenging }(\%)= & ((\text { Blank } \mathrm{AB}-\text { Sample } \mathrm{AB}) / \\
& \text { Blank } \mathrm{AB}) \times 100
\end{aligned}
$$

\subsection{Computational method}

The inclusion complexation between vanillin and these three types of CD (HP $\beta C D, H P \gamma C D$ and $M \beta C D)$ was also studied by computational techniques. We performed $a b$ initio calculations ${ }^{34}$ within the generalized gradient approximation ${ }^{35}$ including a van der Waals correction ${ }^{36}$ implemented in the Vienna $A b$ initio simulation package (VASP). ${ }^{37,38}$ The projector augmented-wave method (PAW) ${ }^{38}$ has been utilized for pseudopotentials of all elements with a plane-wave basis set having a kinetic energy cutoff of $520 \mathrm{eV}$. The initial structures of HP $\beta C D, H P \gamma C D$ and $\mathrm{M} \beta C D$ were obtained from the Cambridge Structural Database. ${ }^{39}$ Firstly, each of $\mathrm{CD}$, vanillin molecule, and their inclusion complex (IC) was relaxed in a vacuum using the conjugate gradient algorithm without any constraints by setting convergence criteria on the total energy and force to $10^{-4} \mathrm{eV}$ and $10^{-2} \mathrm{eV} \AA^{-1}$, respectively. Next, the effect of water on the formation of IC was analyzed by using the implicit solvent model. ${ }^{40}$ Combined with $a b$ initio methods, this model splits the system into an explicit part (solute), which is treated quantum mechanically and an implicit part (solvent), which is treated as a continuum. This solvent model also takes into account dispersive interactions ${ }^{41}$ and is implemented in VASP (VASPsol). ${ }^{42,43}$

\section{Results and discussion}

\subsection{Electrospinning of vanillin/CD-IC NF}

Firstly, IC solutions were prepared by using vanillin and three different modified CD types (HP $\beta C D, H P \gamma C D$ and $M \beta C D)$, then NFs were obtained from these solutions by using the electrospinning technique (Fig. 1a-c). From our previous study, we have determined the optimum concentrations of $\mathrm{HP} \beta C D$, HP $\gamma \mathrm{CD}$ and M $\beta \mathrm{CD}$ in water, DMF and DMAc solvent systems for formation of uniform and bead-free NFs. ${ }^{29}$ Here, we used the same concentrations during the electrospinning of vanillin/CD-IC systems and we have successfully obtained bead-free NFs from all IC solutions, except vanillin/HP $\gamma \mathrm{CD}-\mathrm{IC}$ NFs produced in DMAc (Fig. 2). In addition, as it is observed from Fig. 1c, the ultimate free-standing vanillin/CD-IC NFs can
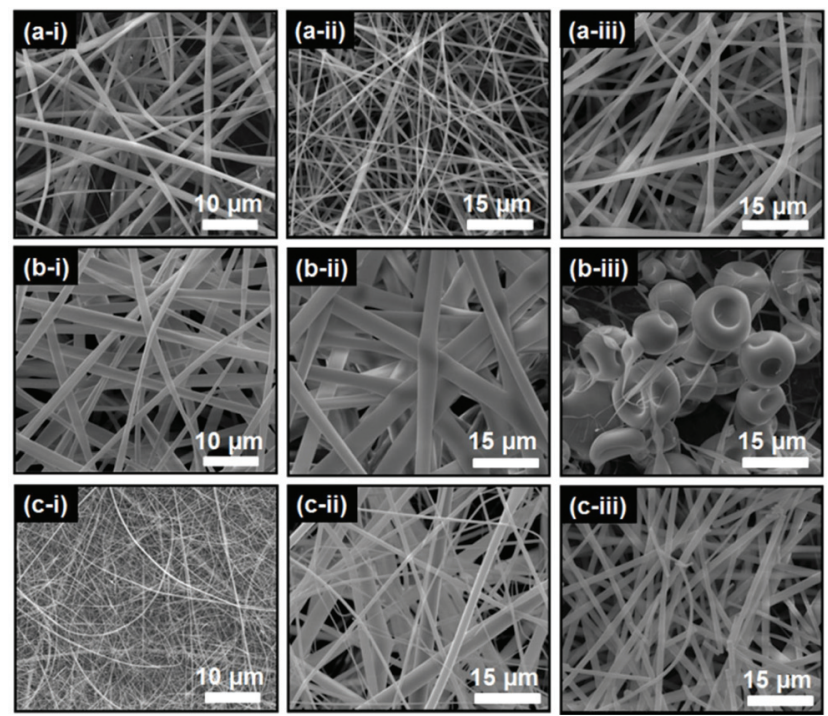

Fig. 2 Representative SEM images of electrospun (a) vanillin/HP $\beta C D-I C$ NF, (b) vanillin/HP $\gamma C D-I C$ NFs and (c) vanillin/M $\beta C D-I C$ NFs obtained in (i) water, (ii) DMF and (iii) DMAc. 
Table 1 Properties of the vanillin/CD-IC solutions and the resulting NFs

\begin{tabular}{|c|c|c|c|c|}
\hline Solutions & $\% \mathrm{CD}(\mathrm{w} / \mathrm{v})$ & Viscosity (Pa s) & Average fiber diameter (nm) & Fiber diameter range (nm) \\
\hline Vanillin/HPßCD-IC-DMF & 120 & 0.3850 & $750 \pm 125$ & $230-1750$ \\
\hline Vanillin/HP $\gamma$ CD-IC-water & 160 & 0.2200 & $1665 \pm 435$ & $565-2980$ \\
\hline Vanillin/HP $\gamma$ CD-IC-DMF & 125 & 0.6130 & $3510 \pm 930$ & $1320-5200$ \\
\hline Vanillin/HP $\gamma$ CD-IC-DMAc & 125 & 1.8500 & - & - \\
\hline Vanillin/MßCD-IC-water & 160 & 0.1600 & $155 \pm 100$ & $50-550$ \\
\hline
\end{tabular}

be obtained as a web along with handy and flexible characteristics.

The properties of the vanillin/CD-IC solutions and the fiber diameter of vanillin/CD-IC NFs are summarized in Table 1. In the case of vanillin/HP $\beta$ CD-IC NFs, the average fiber diameter (AFD) values were calculated as $540 \pm 130 \mathrm{~nm}, 750 \pm 125 \mathrm{~nm}$ and $1450 \pm 310 \mathrm{~nm}$ for water, DMF and DMAc based systems, respectively. On the other hand, the AFD values were determined as $1665 \pm 435 \mathrm{~nm}$ for vanillin/HP $\gamma \mathrm{CD}$-IC-water NFs and $3510 \pm 930 \mathrm{~nm}$ for vanillin/HP $\gamma$ CD-IC-DMF NFs. As is observed, the vanillin/HP $\gamma \mathrm{CD}-\mathrm{IC}$ NFs have significantly higher fiber diameter values compared to vanillin/HPBCD-IC NFs, and this originated from the higher viscosity value of vanillin/HP $\gamma$ CD-IC solutions that leads to less stretching, and hence thicker fiber formation during electrospinning. The more viscous characteristic of $\mathrm{HP} \gamma \mathrm{CD}$ solutions probably arises from the bigger $\mathrm{HP} \gamma \mathrm{CD}$ aggregate size. ${ }^{29}$ In addition, the lower conductivity of the HP $\gamma \mathrm{CD}$ systems compared to $\mathrm{HP} \beta C D$ solutions can be the other effective factor for the thicker HP $\gamma \mathrm{CD}$ fibers. ${ }^{29}$ Even for vanillin/HP $\gamma \mathrm{CD}$-IC prepared in DMAc, we observed bead structures and were not be able to obtain uniform NFs. The reason for this situation can possibly be the extremely high viscosity (Table 1) and low conductivity ${ }^{29}$ of DMAc systems that did not allow the morphology transition completely from beads to the fiber structures because of inadequate stretching during the process. Even so, the characterization of this sample was performed as with other NFs. Because of its beaded feature, it was named as vanillin/HP $\beta$ CD-IC-BNFs.

For vanillin/M $\beta C D-I C$ NFs, we have obtained AFD values of $155 \pm 100 \mathrm{~nm}, 1070 \pm 490 \mathrm{~nm}$ and $1285 \pm 350 \mathrm{~nm}$ for water, DMF and DMAC solvents, respectively. The viscosity of vanillin/M $\beta C D-I C$ solutions is higher than vanillin/HP $\beta C D-I C$ solutions, however, AFDs are not considerably different from each other. This is probably due to the higher conductivity of $\mathrm{M} \beta \mathrm{CD}$ solutions compared to $\mathrm{HP} \beta \mathrm{CD}$ solutions ${ }^{29}$ that eliminates the effect of the higher viscosity level and provides more stretching of the electrospinning jet.

\subsection{Structural characterizations of vanillin/CD-IC NFs}

Here, we have demonstrated the feasibility of CD-IC NF production from three different modified CD types in different solvent systems with vanillin chosen as a flavor/fragrance guest molecule. The detailed structural characterizations were performed for vanillin/CD-IC NFs to investigate the differences between nanofibrous web samples since three types of $\mathrm{CD}$ (HP $\beta C D, H P \gamma C D$ and $\mathrm{M} \beta \mathrm{CD}$ ) and three types of solvent (water, DMF and DMAc) were used for electrospinning.

Initially, FTIR analyses for vanillin/CD-IC NF samples were performed to prove the presence of vanillin and the existence of an interaction between vanillin and $\mathrm{CD}$ molecules. The FTIR spectra which enabled a comparative study between electrospun vanillin/CD-IC NFs and pure vanillin are depicted in Fig. 3. For all vanillin/CD-IC NFs, the prominent absorption peaks observed around 1020, 1070 and $1150 \mathrm{~cm}^{-1}$ correspond to the coupled $\mathrm{C}-\mathrm{C} / \mathrm{C}-\mathrm{O}$ stretching vibrations and the antisymmetric stretching vibration of the $\mathrm{C}-\mathrm{O}-\mathrm{C}$ glycosidic bridge of CD molecules. ${ }^{6,19}$ On the other hand, the FTIR spectrum of pure vanillin exhibited the most significant characteristic peaks at $1300,1510,1592$, and $1666 \mathrm{~cm}^{-1}$ owing to plane aromatic $\mathrm{CH}$ deformation, stretching absorption of the benzene ring and stretching of $\mathrm{C}=\mathrm{O}$ of the aldehyde group in the vanillin structure. ${ }^{6}$ The absorption bands of vanillin at the above ranges $\left(1300,1510,1592\right.$ and $1666 \mathrm{~cm}^{-1}$ ) are also observed for all vanillin/CD-IC NFs and this confirms the existence of vanillin in CD-IC NFs (Fig. 3). Vanillin positions in the hydrophobic cavity in a way that the aldehyde group locates near to the wide end and the phenolic group near to the narrow end of CD (Fig. 1a) $)^{14-16}$ as also confirmed by our molecular modeling study. During this conjugation, the stability of IC is provided by dipolar interactions of the phenyl ring protons with cavity protons, in addition to hydrogen bonds, formed between aldehyde groups of vanillin and secondary hydroxyl groups of $\mathrm{CD} .{ }^{14-16}$ These interactions can cause some shift to the higher or lower wavenumber of vanillin characteristic peaks at the FTIR spectra. ${ }^{6}$ Here, we have detected shifts for characteristic peaks of vanillin, that is, the peak at $1300 \mathrm{~cm}^{-1}$ was shifted to the $1292-1295 \mathrm{~cm}^{-1}$ range and the peak at $1666 \mathrm{~cm}^{-1}$ was shifted to the $1670-1679 \mathrm{~cm}^{-1}$ range, respectively (Fig. 3). These observed shifts proved the formation of an inclusion complex between vanillin and $\mathrm{CD}$ molecules, such that the shifts at $1300 \mathrm{~cm}^{-1}$ originated from the dipolar interaction of the phenyl ring proton with the cavity proton, and the $1666 \mathrm{~cm}^{-1}$ band shift $(\mathrm{C}=\mathrm{O}$ stretching $)$ 

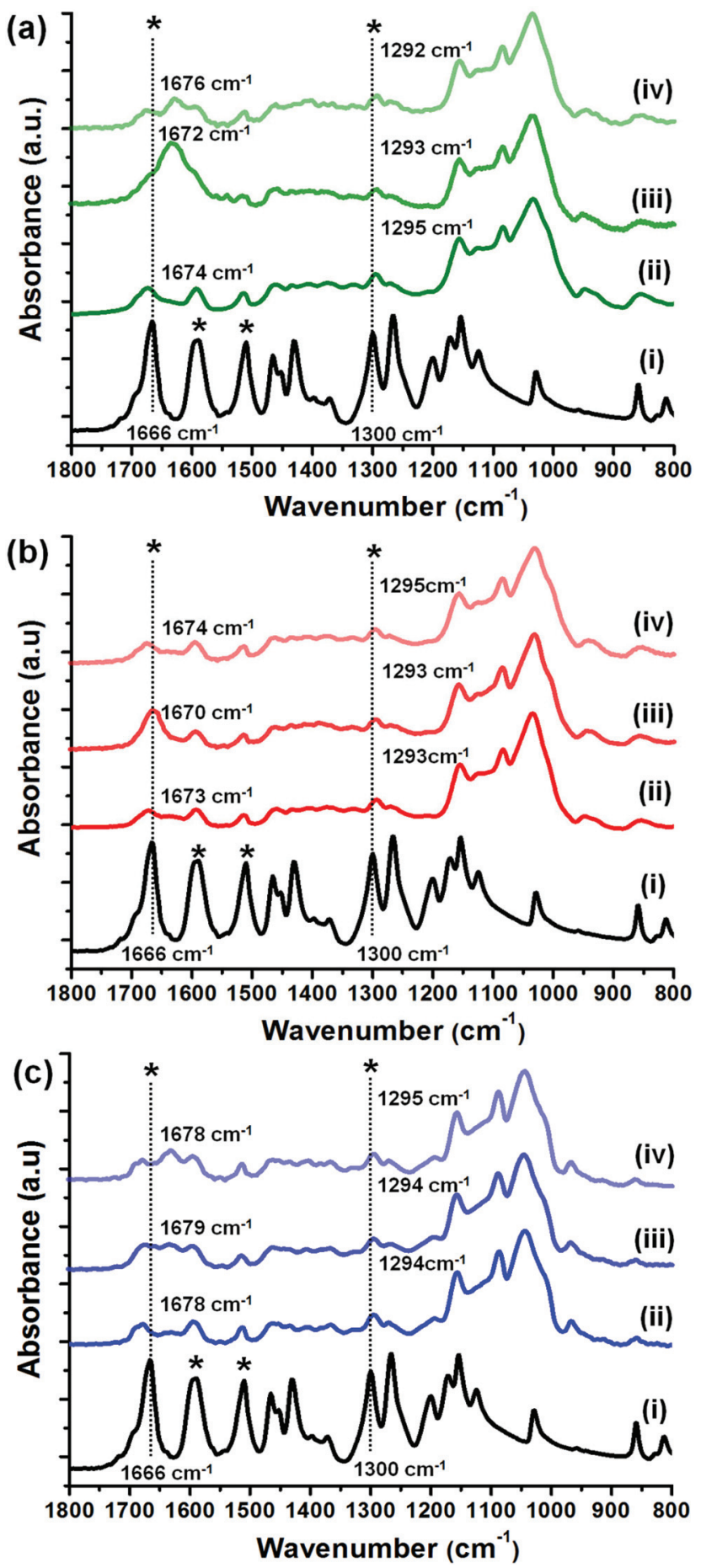

Fig. 3 FTIR spectra of (a) vanillin/HP $\beta C D-I C$ NFs, (b) vanillin/HP $\gamma$ CD-IC NFs and (c) vanillin/M $\beta C D-I C$ NFs obtained in (ii) water, (iii) DMF and (iv) $\mathrm{DMAc}$, as compared with (i) pure vanillin data.

is due to the hydrogen bond formation between secondary hydroxyl groups of $\mathrm{CD}$ and the carbonyl groups of vanillin molecules. ${ }^{15}$

The evidence of inclusion complexation between CD molecules and vanillin was also confirmed using XRD. XRD is a very useful tool for studying the crystalline characteristics of pure vanillin powder and vanillin/CD-IC NF web samples. Actually, vanillin is a crystalline material having a distinct diffraction centered at $2 \theta=13^{\circ}$ (Fig. 4a-c(i)). On the other hand, modified CD is known with their amorphous structure unlike their crystalline native types $\left(\alpha-\mathrm{CD}, \beta-\mathrm{CD}\right.$ and $\gamma$-CD). ${ }^{26,27}$ In modified $\mathrm{CD}$, the random substitution of the hydroxyl groups of CD with methyl or hydroxypropyl groups prevents the particular orientation of $\mathrm{CD}$ molecules and therefore resulted in amorphous material. XRD graphs show that the vanillin/CD-IC NF web samples have amorphous characteristics just like their pristine $\mathrm{HP} \beta \mathrm{CD}, \mathrm{HP} \gamma \mathrm{CD}$ and $\mathrm{M} \beta \mathrm{CD} \mathrm{NFs}^{29}$ without showing any specific vanillin diffraction peak. The absence of a vanillin diffraction peak in vanillin/CD-IC NFs strongly suggested the inclusion complexation between vanillin and $\mathrm{CD}$ molecules ( $\mathrm{HP} \beta \mathrm{CD}, \mathrm{HP} \gamma \mathrm{CD}$ and $\mathrm{M} \beta \mathrm{CD}$ ), as the encapsulation of vanillin molecules inside the CD cavity prevents the formation of vanillin crystals by separating guest molecules from each other. ${ }^{6}$

DSC is also a very practical method to affirm whether the guest molecules are encapsulated in the $\mathrm{CD}$ cavities or not. Hence, DSC measurement was performed as well, to evaluate the IC formation between $\mathrm{CD}$ and vanillin by investigating the thermal behaviour of the vanillin/CD-IC NF web samples. In the case of complexation, the melting or sublimation points of guest molecules would mostly shift or disappear in the DSC thermograms, or these thermal transitions would be noticed if there are any uncomplexed guest molecules in the sample. Fig. $4 \mathrm{~d}-\mathrm{f}$ depicts DSC thermograms of pure vanillin and vanillin/CD-IC NFs. As is clear, the pure vanillin thermogram shows a melting point at $82{ }^{\circ} \mathrm{C}$, conversely, there is no endothermic peak at this point for vanillin/CD-IC NFs confirming the full encapsulation of vanillin molecules in the $\mathrm{CD}$ cavity by complexation.

Additionally, the total enthalpy change $\left(\Delta H\right.$ in $\left.\mathrm{J} \mathrm{g}^{-1}\right)$ observed in the DSC thermograms of vanillin/CD-IC NFs correlate with the dehydration of $\mathrm{CD}$ molecules, and so $\Delta H$ differences between pure CD and CD-IC may also indicate complex formation. $^{44,45}$ During the complexation process, guest compounds compete with water molecules, locating inside the CD cavity, so as expected, by the replacement of water molecules, the $\Delta H$ value of dehydration will be lower for the CD-IC structure compared to the pure CD form. Here, $\Delta H$ and peak temperature assessments were carried out by using TA-Instruments Software and summarized in Table S1. $\dagger$ The obtained enthalpies did not indicate a definite trend in terms of $\mathrm{CD}$ or solvent types, so the results could not be compared exactly with each other. However the apparent decrease for the $\Delta H$ value and increase for peak temperature can be considered as rather informative and these results eloquently supported inclusion complex formation between vanillin and CD molecules for all samples.

TGA is a practical technique to procure information about the volatility and thermal stability of fragrances and flavors. So, the comparative thermal properties of vanillin and vanillin/CD-IC NFs were investigated by this method and TGA thermograms are depicted in Fig. 5. The thermal evaporation 

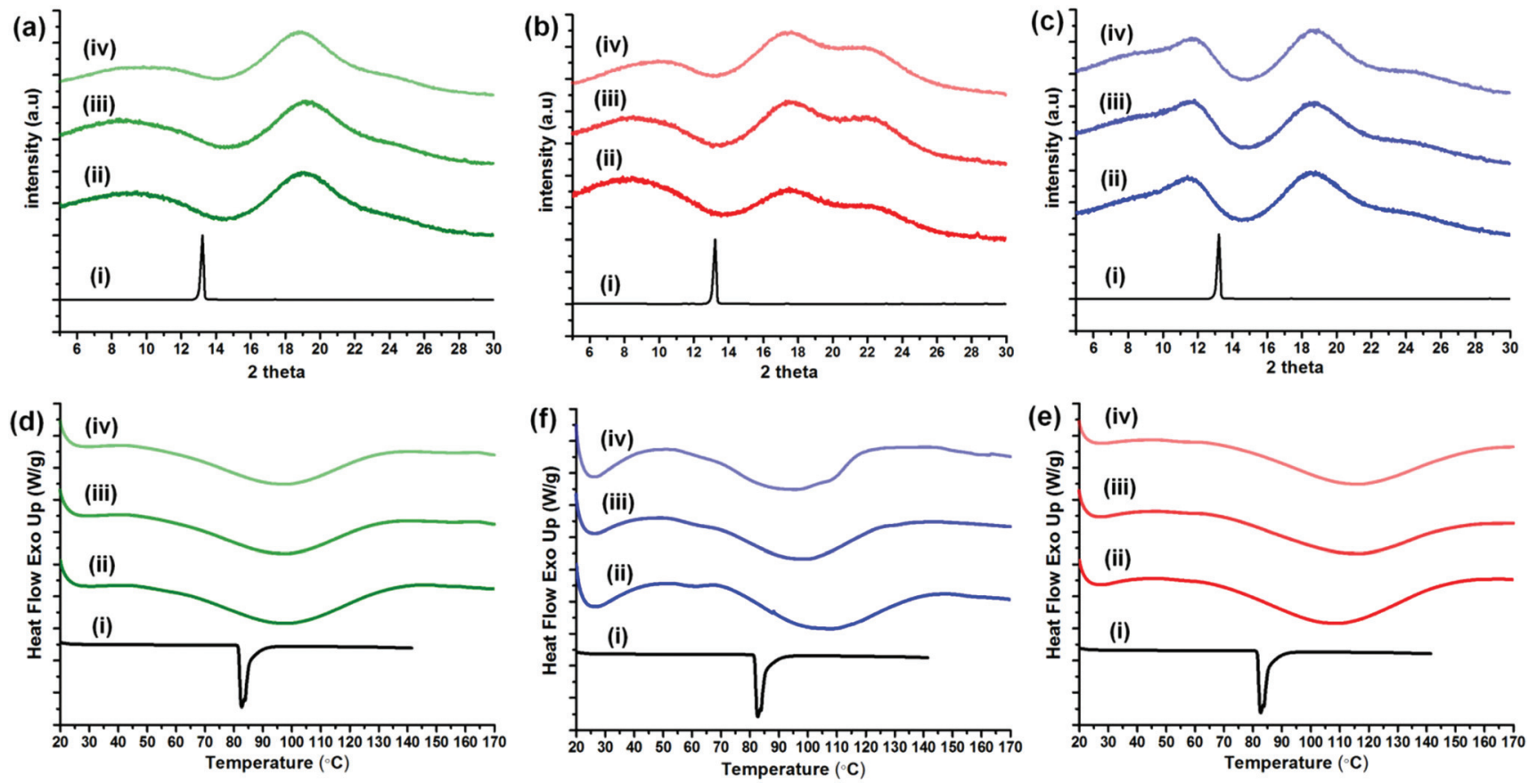

Fig. 4 XRD and DSC graphs of (a and d) vanillin/HP $\beta C D-I C$ NFs, (b and e) vanillin/HP $\gamma C D-I C$ NFs and (c and f) vanillin/M $\beta C D-I C$ NFs obtained in (ii) water, (iii) DMF and (iv) DMAc, as compared to (i) pure vanillin data.
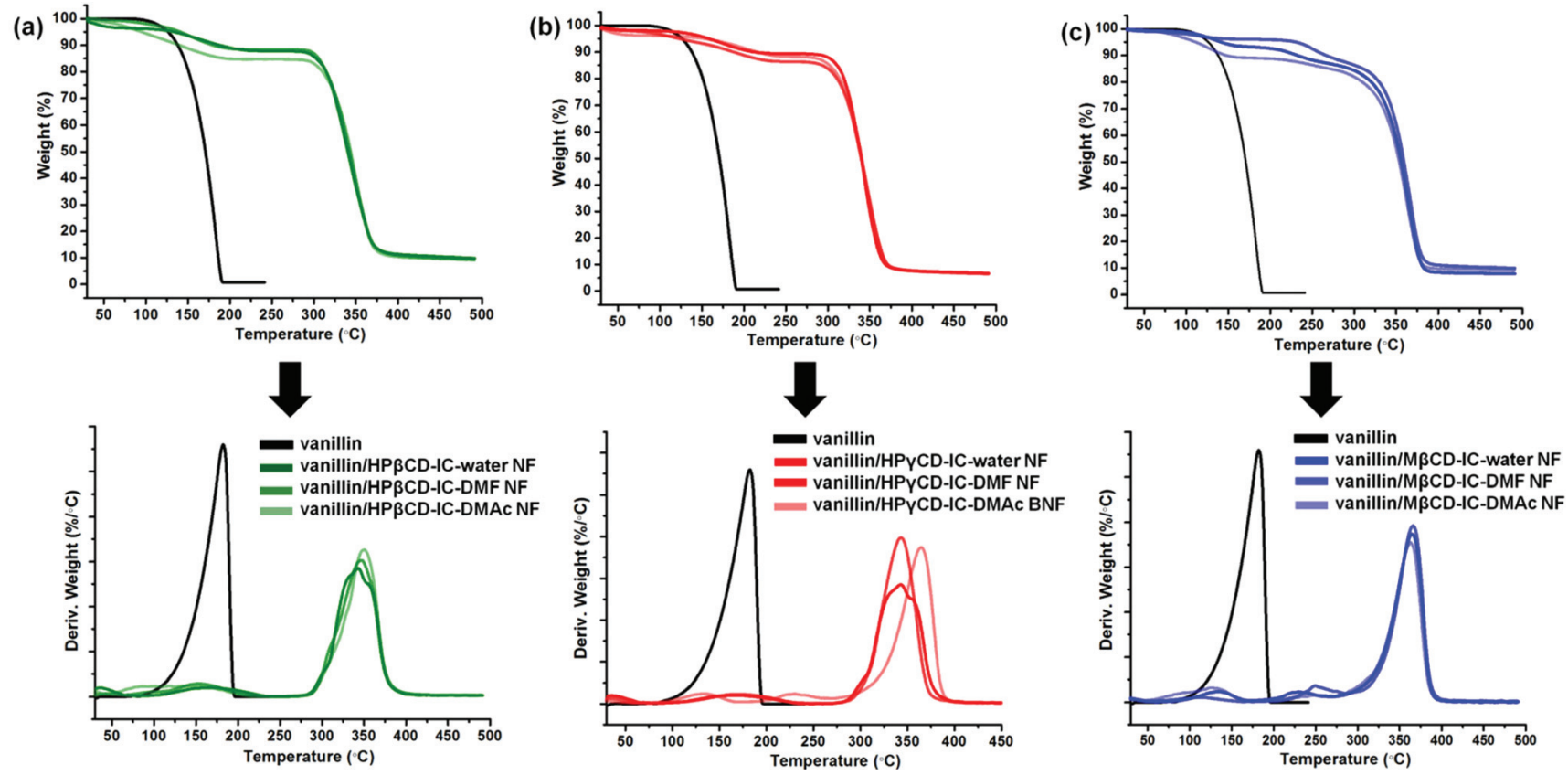

Fig. 5 TGA thermograms and their derivatives for (a) vanillin/HP $\beta C D-I C$ NFs, (b) vanillin/HP $\gamma$ CD-IC NFs and (c) vanillin/M $\beta C D-I C$ NFs, as compared with pure vanillin data.

of vanillin started at about $80^{\circ} \mathrm{C}$ (Td onset) and continued till $195^{\circ} \mathrm{C}$ showing the volatile nature of vanillin. The TGA thermograms of vanillin/CD-IC NFs are composed of three main stages of weight losses between $25{ }^{\circ} \mathrm{C}$ and $400{ }^{\circ} \mathrm{C}$; the initial weight loss below $100{ }^{\circ} \mathrm{C}$ is due to water loss, the second weight loss between 150 and $250{ }^{\circ} \mathrm{C}$ is due to the evaporation of vanillin, and the major weight loss above $300{ }^{\circ} \mathrm{C}$ corresponds to the main thermal degradation of $\mathrm{CD}$ (Fig. 5). As is 
seen from the derivative thermograms, the evaporation of pure vanillin was completed at about $195{ }^{\circ} \mathrm{C}$, however, in the case of vanillin/CD-IC NFs, the evaporation of vanillin was shifted to a higher temperature. Actually, the higher thermal evaporation of vanillin is anticipated for vanillin/CD-IC NFs, confirming that the thermal stability of vanillin was increased due to the strong interaction with the CD cavity by the inclusion complexation.

For vanillin/HP $\beta$ CD-IC NFs and vanillin/HP $\gamma$ CD-IC NFs, the evaporation of vanillin was over at about $240{ }^{\circ} \mathrm{C}$ and 255-260 ${ }^{\circ} \mathrm{C}$, respectively suggesting complex formation between $\mathrm{CD}$ and vanillin. On the other hand, there is an additional weight loss step for vanillin/MBCD-IC NFs when compared to vanillin/HP $\beta$ CD-IC NFs and vanillin/HP $\gamma$ CD-IC NFs (Fig. 5c). Here, the evaporation of vanillin was completed in two successive steps for vanillin/M $\beta C D$-IC NFs; the initial weight loss was nearly same with the vanillin/HPßCD-IC NFs and vanillin/HP $\gamma \mathrm{CD}-\mathrm{IC}$ NFs, yet, the second weight loss was completed at about $270-285^{\circ} \mathrm{C}$. These two evaporation stages of vanillin in vanillin/M $\beta C D$-IC NFs is possibly due to the presence of two different types of interaction between vanillin and CD molecules, in which the second one is much stronger, since the thermal evaporation of complexed vanillin was delayed to a much higher temperature compared to $\mathrm{HP} \beta \mathrm{CD}$ and $\mathrm{HP} \gamma \mathrm{CD}$ systems (Fig. 5a and b). Actually, the TGA technique enables the calculation of the ratio of components in the sample. Unfortunately, overlapping weight losses hinder the calculation of the exact amount of vanillin from TGA thermograms. Nevertheless, the TGA results clearly demonstrated the enhanced thermal stability of vanillin due to the inclusion complexation with $\mathrm{CD}$.

The ${ }^{1} \mathrm{H}$-NMR measurements enable us to determine the molar ratio of guest and host molecules in CD-IC systems. ${ }^{19-23}$ Hence, ${ }^{1} \mathrm{H}$-NMR analyses were carried out by integrating the peak ratio of the characteristic chemical shifts corresponding to vanillin and $\mathrm{CD}$ to calculate the vanillin quantity in vanillin/CD-IC NFs. For vanillin/HP $\beta C D-I C$ NFs and vanillin/ HP $\gamma$ CD-IC NFs, the characteristic peak at about $1.0 \mathrm{ppm}$, and for vanillin/M $\beta$ CD-IC NFs, the peak region at about 4.9-4.7 ppm was used for the calculations. In the case of vanillin, the characteristic peak at $9.8 \mathrm{ppm}$ was taken into account to represent the molar ratio between host and guest molecules in the vanillin/CD-IC NFs (Fig. S1†). The calculated molar ratios of vanillin : $\mathrm{CD}$ are summarized in Table $\mathrm{S} 1 \dagger$ and there are expressive differences between samples depending on the $\mathrm{CD}$ types used for complexation and the solvent types used for electrospinning. As seen from the table, molar ratios (vanillin : CD) determined by ${ }^{1} \mathrm{H}$-NMR measurements are a little bit lower compared to their initial molar ratio (vanillin:CD; $\sim 1: 1)$ possibly because of the evaporation of uncomplexed vanillin during the electrospinning process or during storage. Here, ${ }^{1} \mathrm{H}-\mathrm{NMR}$ results revealed that vanillin/HPßCD-IC NFs and vanillin/HP $\gamma$ CD-IC NFs have almost the same complexation efficiency (molar ratio-vanillin : $\mathrm{CD} ; \sim 0.40-0.77: 1$ ), on the other hand, vanillin/M $\beta C D-I C$ NFs have shown a higher molar ratio (vanillin: CD; $\sim 0.93-1.00: 1$ ) among other samples
(Table S1 $\uparrow$ ). Therefore, the structural differences, originating from the modifications, come into prominence in this situation and most probably, steric hindrance of the hydroxypropyl- group of $\mathrm{HP} \beta \mathrm{CD}$ and $\mathrm{HP} \gamma \mathrm{CD}$ inhibited entry of the vanillin molecule into the $\mathrm{CD}$ cavity, which is not the case for $\mathrm{M} \beta \mathrm{CD} .{ }^{46,47}$ Furthermore, the introduction of large substituents (such as hydroxypropyl), could lead to axial elongation at the $\mathrm{CD}$ torus during chemical modification, ${ }^{11}$ so $\mathrm{M} \beta \mathrm{CD}$ might represent a more favorable and rigid environment for vanillin molecules compared to HP $\beta C D$ along with a better size fit. This also correlates with our findings from our computational modeling study as discussed in the below section.

Correspondingly, both experimental and theoretical data suggested that $\mathrm{M} \beta \mathrm{CD}$ was able to preserve vanillin more efficiently by inclusion complexation when compared to $\mathrm{HP} \beta \mathrm{CD}$ and $\mathrm{HP} \gamma \mathrm{CD}$. Hence, a much lower amount of vanillin was lost for the vanillin/M $\beta C D-I C$ NFs during the electrospinning process when compared to vanillin/HP $\beta C D-I C$ NFs and vanillin/HP $\gamma$ CD-IC NFs. Another point which should be noted is that, depending on the solvent type used, significant differences were observed between initial and final vanillin: CD molar ratios especially in the case of DMF and DMAc systems (Table S1†). So, when the solvent effect is evaluated, for vanillin/HP $\beta$ CD-IC NF, the molar ratio of vanillin with respect to HPßCD (vanillin: $\mathrm{CD}$ ) is higher for vanillin/ HP $\beta C D-I C-w a t e r$ NFs $(0.75: 1)$ compared to vanillin/ HP $\beta C D-I C-D M F$ NFs $(0.63: 1)$ and it is at the lowest value for vanillin/HPßCD-IC-DMAc NFs $(0.55: 1)$. The same trend was also seen for $\mathrm{HP} \gamma \mathrm{CD}$ samples and the molar ratios were calculated in the order of vanillin/HP $\gamma \mathrm{CD}-\mathrm{IC}$ water NFs $(0.77: 1)>$ vanillin/HP $\gamma$ CD-IC-DMF NFs $(0.72: 1)>$ vanillin/HP $\gamma$ CD-IC-DMAc NFs $(0.40: 1)$. In the case of

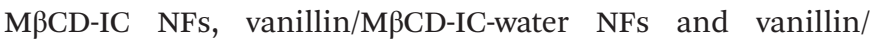
MBCD-IC-DMF NFs have the same molar ratio level $(1.00: 1)$ with minimal lost, however, a higher loss was observed for vanillin/M $\beta$ CD-IC-DMAc NF $(0.93: 1)$. Comparing the solvent systems for electrospinning of vanillin/CD-IC, the preservation of vanillin in CD-IC was in the order of water $>$ DMF $>$ DMAc.

Actually, this is anticipated since the complex formations become significantly more effective in aqueous medium for non-polar guest molecules owing to the reduction of repulsive interactions between the hydrophobic guest and the aqueous environment. ${ }^{10}$ On the other hand, the non-polar nature of DMF and DMAc make them more attractive solvents for the solubility of vanillin, so entering into the CD cavity in these solvents is not preferable as the water environment results in lower complexation efficiency. Moreover, for vanillin/ HP $\gamma$ CD-IC-DMAc-BNF, the loss of vanillin was higher compared to vanillin/HP $\beta C D$-IC-DMAc-NFs, suggesting that the fiber structure was better than beaded structures for the preservation of vanillin molecules in CD-IC systems.

For further studies, vanillin/CD-IC NF samples electrospun from the water based system were selected to investigate the water solubility and antioxidant property of these webs which possibly will be of importance for food based applications. In 
addition, computational modeling studies were carried out to investigate the solvation and inclusion complexation energy differences according to CD types.

\subsection{Molecular modeling for vanillin/CD-IC}

Complexation reactions of the guest molecule within $\mathrm{HP} \beta C D$, $\mathrm{HP} \gamma \mathrm{CD}$ and $\mathrm{M} \beta \mathrm{CD}$ are entirely governed by the interactions between guest and host molecules, and these interactions could be altered in the solvent. Therefore, we carried out structural optimization of vanillin, CD and their possible IC in a vacuum, followed by optimizations in water simulating the effect of the solvent environment. To form IC, single vanillin is introduced into the cavity of $\mathrm{HP} \beta \mathrm{CD}, \mathrm{HP} \gamma \mathrm{CD}$, and $\mathrm{M} \beta \mathrm{CD}$ at various possible locations with different orientations of the guest molecule. The path through the wide and the narrow rim of $\mathrm{CD}$ and the possible orientations of vanillin (head(hydroxyl $(\mathrm{OH})$ and methoxy $\left(\mathrm{OCH}_{3}\right.$ group)) or tail-(aldehyde (CHO) group)) are tested. Our calculations indicate that the vanillin molecule prefers to stay in the cavity of $\mathrm{CD}$ with its hydroxyl and methoxy groups headed towards the narrow rim, keeping the polar functional group $(\mathrm{OH})$ outwards. Among all the possibilities, the complexation energy $\left(E_{\text {comp }}\right)$ is calculated for the most favorable energy configurations which are shown in Fig. 6 by using the formula:

$$
E_{\text {comp }}=E_{\mathrm{CD}}+E_{\text {guest }}-E_{\mathrm{IC}}
$$

where $E_{\mathrm{CD}}, E_{\text {guest }}$, and $E_{\mathrm{IC}}$ is the total energy of CD (HP $\beta C D$, $\mathrm{HP} \gamma \mathrm{CD}$, or M $\beta C D$ ), the guest vanillin molecule and their IC, respectively. The obtained results in 1:1 stoichiometry for the water environment are summarized in Table 2. The results point out that the complexation reaction occurs in a water medium with varying $E_{\text {comp }}$ depending on the CD type. The complexation energies ranged in the order of M $\beta C D>$ HP $\beta C D$ $>\mathrm{HP} \gamma \mathrm{CD}$ which means that the strongest and weakest IC belongs to the M $\beta C D$ and HP $\gamma \mathrm{CD}$ systems, respectively. Actually, modeling results are in agreement with our ${ }^{1} \mathrm{H}-\mathrm{NMR}$ results. Moreover, the modeling study also revealed that the apolar interaction between methyl groups of $\mathrm{M} \beta \mathrm{CD}$ and vanillin molecules might slightly enhance the complexation strength. This stronger interaction could explain the higher thermal evaporation of vanillin in the case of vanillin/M $\beta C D-I C$ NFs which was observed in the TGA thermograms (Fig. 5). We also calculated the solvation energy of the vanillin/CD-IC systems in water to estimate their solubility. The solvation energy $\left(E_{\text {solv }}\right)$ in water can be calculated as:

$$
E_{\text {solv }}=E_{\text {water }}-E_{\text {vacuum }}
$$

where $E_{\text {water }}$, and $E_{\text {vacuum }}$ is the total energy of vanillin, CD, or IC in solvent and vacuum, respectively. Although $E_{\text {solv }}$ does not indicate the absolute solubility, solubility trends can still be deduced. The calculated $E_{\text {solv }}$ for IC in water are listed in Table 2. Accordingly, while vanillin/HP $\gamma \mathrm{CD}-\mathrm{IC}$ has the highest solubility $\left(-82.5 \mathrm{kcal} \mathrm{mol}^{-1}\right)$, vanillin/M $3 \mathrm{CD}$-IC has the lowest solubility $\left(-29.0 \mathrm{kcal} \mathrm{mol}^{-1}\right)$ in water and these findings are also compatible with the water solubility trend of pure $\mathrm{HP} \beta \mathrm{CD}$, HP $\gamma C D$ and M $\beta C D$ mentioned in the literature. ${ }^{11} E_{\text {solv }}$ of single (a)

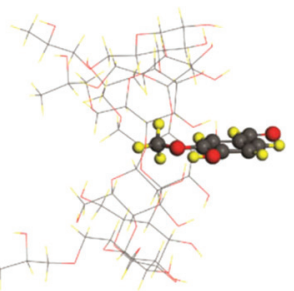

(b)

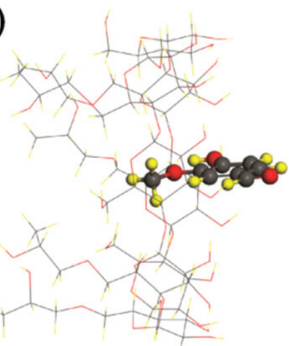

(c)

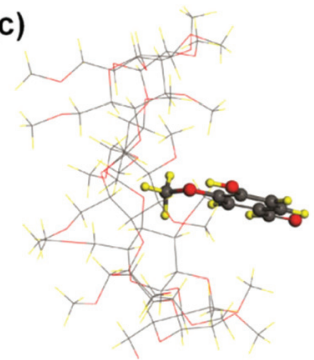

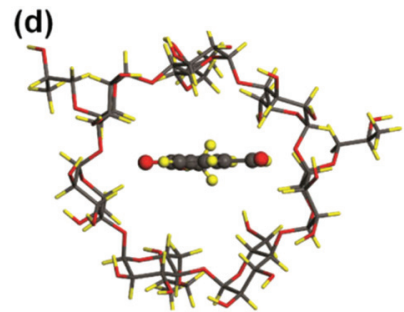

(e)

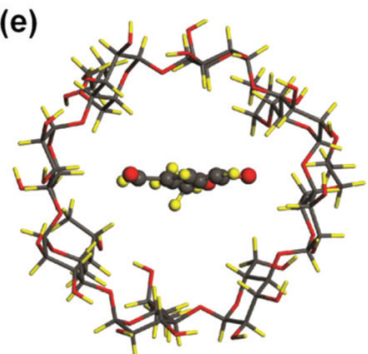

(f)

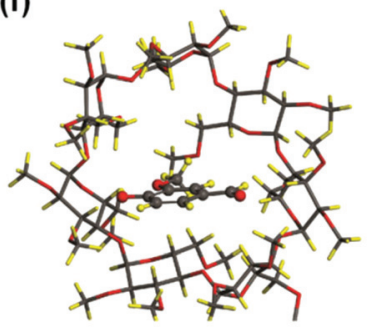

Fig. 6 The side view $(a-e)$ and the front view through a narrow rim $(b-f)$ of IC of vanillin within $H P \beta C D, H P \gamma C D$, and M $\beta C D$. The hydroxyl and methoxy side groups of vanillin headed towards the narrow rim. (Gray, red and yellow spheres represent carbon, oxygen and hydrogen atoms, respectively.)

Table 2 Complexation and solvation energies of the vanillin molecule within $\mathrm{HP} \beta C D, H P \gamma C D$ and $M \beta C D$ in water

\begin{tabular}{llll}
\hline Host & Guest & $E_{\text {comp }}^{\text {water }}\left(\mathrm{kcal} \mathrm{mol}^{-1}\right)$ & $E_{\text {solv }}\left(\mathrm{kcal} \mathrm{mol}^{-1}\right)$ \\
\hline HP $\beta C D$ & Vanillin & 6.6 & -75.4 \\
HP $\gamma$ CD & Vanillin & 5.3 & -82.5 \\
M CD & Vanillin & 6.9 & -29.0
\end{tabular}

vanillin in water is $-6.8 \mathrm{kcal} \mathrm{mol}^{-1}$ and that indicates the low solubility of this hydrophobic compound. Our calculations suggest that when IC is more soluble in water, $E_{\text {comp }}$ decreases for all considered types of CD.

\subsection{Phase solubility study and water solubility of vanillin/CD-IC NFs}

Vanillin is a widely used compound in the food industry as a flavor/fragrance agent. However, its solubility would be considered limited depending on the purpose and this would be a drawback for its use. Here, in addition to thermal property enhancement, the solubility of vanillin is also improved by forming IC with CD molecules. Phase solubility diagrams 
(a)
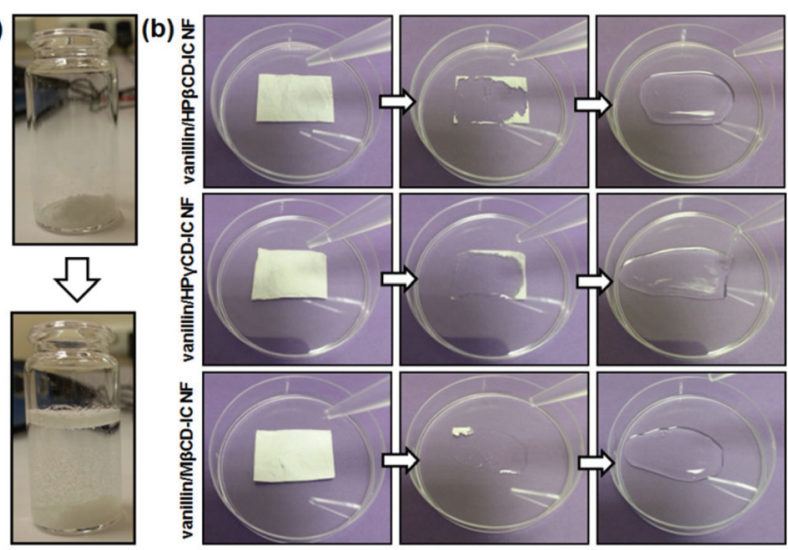

Fig. 7 Presentation of the solubility behavior of (a) pure vanillin and (b) vanillin/HP $\beta C D-I C N F s$, vanillin/HP $\gamma C D-I C N F$ and vanillin/M $\beta C D-I C$ $\mathrm{NFs}$ in water.

belonging to vanillin/CD-IC exhibited an AL type pattern which infers a linear increment in the solubility of vanillin with increasing CD concentration (Fig. S2 $\dagger$ ). Additionally, this linear trend displayed a 1:1 complex formation tendency of vanillin and $\mathrm{CD}$ molecules.

On the other hand, the solubility of the vanillin molecules which were encapsulated in CD-IC NFs was determined by the HPLC technique. For this purpose, the pure vanillin and the CD-IC NFs, which include the same concentration $\left(20 \mathrm{mg} \mathrm{mL}{ }^{-1}\right.$ ) of vanillin, were dissolved in water. While the vanillin/CD-IC NFs were dissolved in water immediately, there were still undissolved vanillin particles in the pure vanillin solution even after stirring overnight (Fig. 7). The HPLC results of the filtered solution of pure vanillin and vanillin/CD-IC NFs demonstrated that, in the case of pure vanillin, the solubility value of $10.2 \pm 0.02 \mathrm{mg} \mathrm{mL}^{-1}$ correlated with the literature limit. ${ }^{16}$ On the other hand, the solubility of vanillin encapsulated in vanillin/CD-IC NFs was determined as $20.6 \pm 0.2,21.6 \pm 0.7$ and $20.4 \pm 0.2 \mathrm{mg} \mathrm{mL}{ }^{-1}$ for vanillin/HP $\beta C D-I C$ NFs, vanillin/HP $\gamma$ CD-IC NFs and vanillin/ MBCD-IC NFs, respectively. These results revealed that we have obtained a complete dissolution for vanillin that we used during the test and the water solubility of vanillin can be significantly improved owing to inclusion complexation with CD.

\subsection{Antioxidant property of vanillin/CD-IC NFs}

The consumption of vanillin is common in food and pharmaceutical industries due to its flavoring potential, however the antimicrobial, anticarcinogenic and antimutagenic properties of vanillin have also widened its use in these applications. Thanks to the phenolic structure, vanillin molecules might also exhibit an antioxidant property, nevertheless, studies on the antioxidant property of vanillin have not provided consistent results because of its lower antioxidant efficiency when compared to its other derivative ( $o$-vanillin, 4-hydroxybenzyl alcohol, 4-hydroxy-3-methoxybenzyl alcohol etc.). ${ }^{5,48-50}$

Here, we investigated the antioxidant property of vanillin/ CD-IC NFs, and we have anticipated obtaining an enhanced antioxidant efficiency due to inclusion complexation when compared to given reports in the literature. ${ }^{5,48-50}$ For this, the antioxidant property of vanillin/CD-IC NFs and pure vanillin was evaluated via the model organic radical, DPPH. This approach is based on the reduction of the DPPH molecule by a hydrogen donor, such as a free radical scavenging antioxidant. Depending on the DPPH reduction, a decrease occurs of the strongest absorption $(517 \mathrm{~nm})$ band and the purple color of solution turns yellowish upon completion of reaction. Fig. 8 displays UV-Vis absorption graphs for pure vanillin, time dependent \%DPPH radical scavenging graphs of vanillin/ CD-IC NFs and photographs of the solutions for all
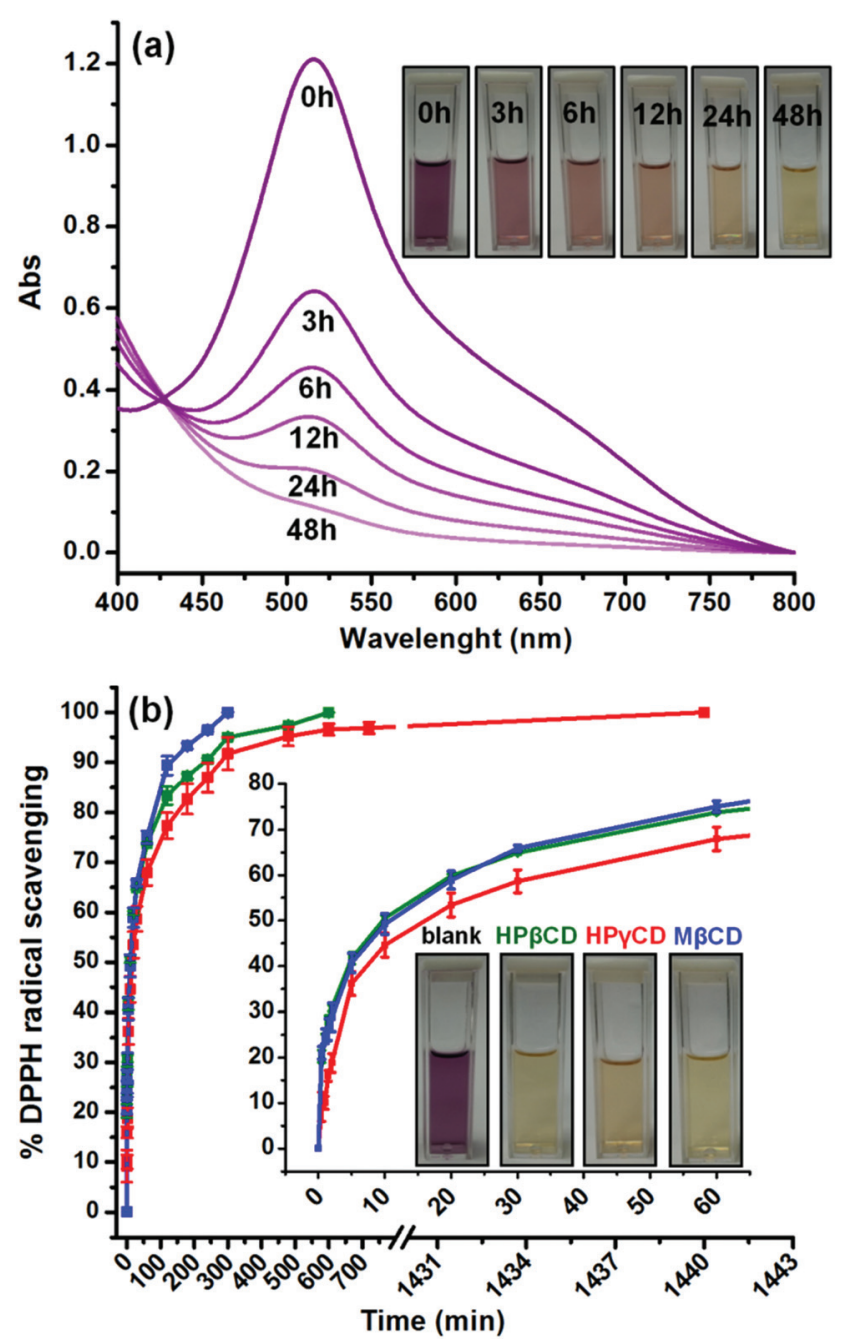

Fig. 8 (a) UV-Vis absorption graphs and the photographs of the solution, indicating the DPPH scavenging efficiency of pure vanillin at progressing time intervals. (b) Time dependent \%DPPH radical scavenging graphs of vanillin/CD-IC NFs and photographs of the final solutions following completion of the reactions. 
systems. Here, the antioxidant tests were carried out by using $12 \mathrm{mg} \mathrm{mL} \mathrm{m}^{-1}$ concentrated clear vanillin/CD-IC NF solutions for all $\mathrm{CD}$ types. On the other hand, the vanillin system was prepared from the highest vanillin initial amount $(12 \% \mathrm{w} / \mathrm{w}$, with respect to the total sample amount) used for $\mathrm{M} \beta \mathrm{CD}$ and the undissolved part was filtered just before the antioxidant experiment.

For both pure vanillin powder and vanillin/CD-IC NFs, phenolic $\mathrm{OH}$ groups of vanillin are responsible for the antioxidant property. However, the conversion of vanillin to its oxidized form (Van-OH to Van- ${ }^{*}, \mathrm{H}^{+}$) requires high energy and that diminishes the radical scavenging potential of this compound. ${ }^{50}$ On the other hand, the enhancement attained with the inclusion complexation is the first conspicuous point in these antioxidant tests. As is observed from Fig. 8, while DPPH radical scavenging was completed at $48 \mathrm{~h}$ in the case of pure vanillin, it took $6 \mathrm{~h}, 10 \mathrm{~h}$ and $24 \mathrm{~h}$ for vanillin/M $\beta \mathrm{CD}-\mathrm{IC}$, vanillin/HP $\beta C D-I C$ and vanillin/HP $\gamma$ CD-IC NFs, respectively. The enhanced antioxidant efficiency of vanillin originated from the IC mechanism which increases the solubility and thus a higher amount of vanillin could take part in the scavenging process. Additionally, interactions existing in the IC structure might be another reason for the improved antioxidant property.

As is explained in the previous studies, the methoxy group $\left(\mathrm{OCH}_{3}\right)$ of vanillin creates steric hindrance during encapsulation into the $\mathrm{CD}$ cavity thus, the $\mathrm{OH}$ group, taking part at the ortho position according to $\mathrm{OCH}_{3}$, could not get close to the primary $\mathrm{OH}$ of $\mathrm{CD}$ to form an interaction. ${ }^{8,15}$ Here, this orientation and the $\mathrm{OH}$ group location out of the $\mathrm{CD}$ cavity were also shown with the modeling study. Owing to this coordination, one might consider that there is no restriction originating from complex formation that could hinder $\mathrm{OH}$ group activity and thus the radical scavenging property of vanillin. However, in our case, the substituents of modified CD could soften steric hindrance occurring at the primary side of $\mathrm{CD}$ and reduce the tension on the $\mathrm{OH}$ group of vanillin molecules. Associated with this, the hydroxyl of vanillin would be stretched and oxidized easier, so reaction with DPPH could be more efficient compared to its uncomplexed form.

The graphs of DPPH scavenging obviously display antioxidant activity variations between vanillin/CD-IC samples (Fig. 8b). The inset graph drawn until $60 \mathrm{~min}$ indicated that vanillin/M $\beta$ CD-IC NFs and vanillin/HP $\beta$ CD-IC NFs have almost the same scavenging rate in the first hour, however vanillin/M $\beta C D-I C$ NFs completed the reaction faster than vanillin/HP $\beta$ CD-IC NFs, most probably due to the higher vanillin content of vanillin/M $\beta \mathrm{CD}-\mathrm{IC}$ NFs as calculated from the ${ }^{1} \mathrm{H}-\mathrm{NMR}$ measurement $(10.4 \%$, w/w and $7.6 \%$, w/w for vanillin/M $\beta$ CD-IC and vanillin/HP $\beta$ CD-IC NFs, respectively). Additionally, vanillin/HP $\gamma$ CD-IC NFs showed the slowest antioxidant activity because of the lowest vanillin content (6.9\%, w/w) among other samples.

Moreover, the binding strength variations depending on the CD type might be another reason for the different anti- oxidant efficiency. As the binding between $\mathrm{CD}$ and vanillin strengthen, the strain on vanillin $\mathrm{OH}$ would be reduced more and it would be oxidized easier to indicate its reduction potential. For vanillin/M $\beta$ CD-IC NFs, the better antioxidant efficiency could be the reason for the possible apolar interaction between the methyl group of $\mathrm{M} \beta \mathrm{CD}$ and the methoxy group of vanillin which was also revealed in the modeling study. The methoxy moiety $\left(\mathrm{OCH}_{3}\right)$, which is primarily responsible for steric hindrance during complexation, would be eliminated easier in vanillin/M $\beta$ CD-IC NFs and more efficient radical scavenging would be obtained by this way. Hereby, we have improved the antioxidant efficiency of vanillin by means of CD-IC formation for electrospun vanillin/CD-IC NF samples.

\section{Conclusions}

To conclude, the electrospinning of vanillin/CD-IC NFs was achieved without using a carrier polymeric matrix. The IC solutions were prepared by using three CD types (HP $\beta C D, H P \gamma C D$ and $\mathrm{M} \beta \mathrm{CD}$ ) in three different solvent systems (water, DMF and DMAc). Except for the vanillin/HP $\gamma$ CD-IC-DMAc system, all vanillin/CD-IC NFs were obtained with uniform fiber morphology. FTIR, XRD, DSC and TGA analyses revealed the existence of vanillin in NFs and IC formation between vanillin and $\mathrm{CD}$ (HP $\beta \mathrm{CD}, \mathrm{HP} \gamma \mathrm{CD}$ and $\mathrm{M} \beta \mathrm{CD})$. Depending on the solvent and CD types used, a significant amount of vanillin was preserved due to inclusion complex formation after electrospinning. The maximum preservation of vanillin was observed for vanillin/M $\beta$ CD-IC NFs (up to $\sim 85 \%$, w/w) and slightly less preservation of vanillin was observed for vanillin/HP $\beta$ CD-IC NFs and vanillin/HP $\gamma$ CD-IC NFs $(\sim 75 \% \mathrm{w} / \mathrm{w})$. On the contrary, our recent study had shown that vanillin could not be well preserved when encapsulated in polyvinyl alcohol (PVA) polymeric electrospun nanofibers without CD-IC. ${ }^{19}$ Moreover, we were able to achieve much higher vanillin loading ( $9 \%$ to $12 \%$, $\mathrm{w} / \mathrm{w}$ ) by electrospinning of polymer-free CD/vanillin-IC-NFs when compared to electrospun polymeric nanofibers containing CD/vanillin-IC $(\sim 5 \%, \mathrm{w} / \mathrm{w}) .{ }^{19}$

Afterwards, vanillin/CD-IC NFs produced in water were chosen for the solubility and antioxidant test, since water based systems are more suitable for food applications owing to the non-toxic nature of the solvent. Computational modeling findings were consistent with the experimental data and revealed that the complexation strengths range in the order of $\mathrm{M} \beta \mathrm{CD}>\mathrm{HP} \beta \mathrm{CD}>\mathrm{HP} \gamma \mathrm{CD}$ and solvation of vanillin is in the order of $\mathrm{M} \beta \mathrm{CD}<\mathrm{HP} \beta \mathrm{CD}<\mathrm{HP} \gamma \mathrm{CD}$. Our results indicated that, while enhanced thermal stability was provided for the vanillin compound, phase solubility studies displayed that the water solubility of vanillin can be linearly enhanced by increasing CD concentration due to inclusion complexation. The water solubility test also suggested the improved solubility of this compound in the case of vanillin/CD-IC NFs.

Moreover, it has been shown that the poor antioxidant property of vanillin can be enhanced with the help of complex 
formation. Briefly, we have produced CD-IC NFs by using FDA approved modified $\mathrm{CD}^{10}$ and the vanillin compound which is widely used as a food ingredient for flavor/fragrance. Hence, the vanillin/CD-IC NFs would be rather attractive in food applications along with the non-toxic nature of $\mathrm{CD}$, the high surface area of electrospun NFs and the improved antioxidant activity/ solubility of vanillin. Moreover, vanillin/CD-IC was turned into a more applicable NF form by electrospinning, compared to its powder state along with having a handy and flexible structure.

\section{Acknowledgements}

The Scientific and Technological Research Council of Turkey (TUBITAK, project\#213M185 and \#113Y348) is acknowledged for funding the research. T. U. and E. D. thank The Turkish Academy of Sciences - Outstanding Young Scientists Award Program (TUBA-GEBIP) for partial funding. A. C. thanks TUBITAK-BIDEB and TUBITAK (project \#113Y348) for the PhD scholarship and postdoctoral fellowship, respectively. The calculations were performed at TUBITAK ULAKBIM, High Performance and Grid Computing Center (TR-Grid eInfrastructure).

\section{Notes and references}

1 J. E. Galgani, B. Nunez and L. A. Videla, Food Funct., 2012, 3, 1319-1323.

2 Y. Shen, B. Hu, B. X. Chen, Q. Miao, C. Wang, Z. Zhu and C. Han, J. Agric. Food Chem., 2014, 62, 10881-10888.

3 Z. Dong, F. Gu, F. Xu and Q. Wang, Food Chem., 2014, 149, 54-61.

4 H. Liang, Q. Yuan, F. Vriesekoop and F. Lv, Food Chem., 2012, 135, 1020-1027.

5 A. Tai, T. Sawano, F. Yazama and H. Ito, Biochim. Biophys. Acta, Gen. Subj., 2011, 1810, 170-177.

6 F. Kayaci and T. Uyar, J. Agric. Food Chem., 2011, 59, $11772-$ 11778.

7 S. Y. Hundre, P. Karthik and C. Anandharamakrishnan, Food Chem., 2015, 174, 16-24.

8 V. Karathanos, V. I. Mourtzinos, K. Yannakopoulou and N. K. Andrikopoulos, Food Chem., 2007, 101, 652-658.

9 J. Szejtli, Chem. Rev., 1998, 98, 1743-1754.

10 E. M. Del Valle, Process Biochem., 2004, 39, 1033-1046.

11 L. Szente and J. Szejtli, Adv. Drug Delivery Rev., 1999, 36, $17-28$.

12 A. Kant, R. S. Linforth, J. Hort and A. J. Taylor, J. Agric. Food Chem., 2004, 52, 2028-2035.

13 M. V. Rekharsky and Y. Inoue, Chem. Rev., 1998, 98, 18751918.

14 S. Divakar, J. Agric. Food Chem., 1990, 38, 940-944.

15 Z. Zeng, Y. Fang and H. Ji, Flavour Fragrance J., 2012, 27, 378-385.

16 R. Ferrazza, B. Rossi and G. Guella, J. Phys. Chem. B, 2014, 118, 7147-7155.
17 S. Ramakrishna, K. Fujihara, W.-E. Teo, T.-C. Lim and Z. Ma, An introduction to electrospinning and nanofibers, World Scientific, 2005.

$18 \mathrm{~J}$. H. Wendorff, S. Agarwal and A. Greiner, Electrospinning: materials, processing, and applications, John Wiley \& Sons, 2012.

19 F. Kayaci and T. Uyar, Food Chem., 2012, 133, 641-649.

20 F. Kayaci, Y. Ertas and T. Uyar, J. Agric. Food Chem., 2013, 61, 8156-8165.

21 F. Kayaci, H. S. Sen, E. Durgun and T. Uyar, Food Res. Int., 2014, 62, 424-431.

22 F. Kayaci, O. C. Umu, T. Tekinay and T. Uyar, J. Agric. Food Chem., 2013, 61, 3901-3908.

23 Z. Aytac, S. Y. Dogan, T. Tekinay and T. Uyar, Colloids Surf., $B, 2014,120,125-131$.

24 Z. Aytac, H. S. Sen, E. Durgun and T. Uyar, Colloids Surf., B, 2015, 128, 331-338.

25 Z. Aytac, S. I. Kusku, E. Durgun and T. Uyar, Mater. Sci. Eng., C, 2016, 63, 231-239.

26 A. Celebioglu and T. Uyar, J. Colloid Interface Sci., 2013, 404, 1-7.

27 A. Celebioglu and T. Uyar, RSC Adv., 2013, 3, 22891-22895.

28 A. Celebioglu and T. Uyar, Chem. Commun., 2010, 46, 69036905.

29 A. Celebioglu and T. Uyar, Nanoscale, 2012, 4, 621-631.

30 A. Celebioglu, H. S. Sen, E. Durgun and T. Uyar, Chemosphere, 2016, 144, 736-744.

31 A. Celebioglu and T. Uyar, Langmuir, 2011, 27, 6218-6226.

32 A. Celebioglu, O. C. Umu, T. Tekinay and T. Uyar, Colloids Surf., B, 2014, 116, 612-619.

33 T. Higuchi and A. Connors, Adv. Anal. Chem. Instrum., 1965, 4, 117-212.

34 W. Kohn and L. J. Sham, Phys. Rev., 1965, 140, A1133.

35 J. P. Perdew, J. Chevary, S. Vosko, K. A. Jackson, M. R. Pederson, D. Singh and C. Fiolhais, Phys. Rev. B: Condens. Matter, 1992, 46, 6671.

36 S. Grimme, J. Comput. Chem., 2006, 27, 1787-1799.

37 G. Kresse and J. Furthmüller, Phys. Rev. B: Condens. Matter, 1996, 54, 11169.

38 P. E. Blöchl, Phys. Rev. B: Condens. Matter, 1994, 50, 17953.

39 F. H. Allen, Acta Crystallogr., Sect. B: Struct. Sci., 2002, 58, 380-388.

40 J. L. Fattebert and F. Gygi, Int. J. Quantum Chem., 2003, 93, 139-147.

41 O. Andreussi, I. Dabo and N. Marzari, J. Chem. Phys., 2012, 136, 064102.

42 S. Petrosyan, A. Rigos and T. Arias, J. Phys. Chem. B, 2005, 109, 15436-15444.

43 K. Mathew, R. Sundararaman, K. Letchworth-Weaver, T. Arias and R. G. Hennig, J. Chem. Phys., 2014, 140, 084106.

44 M. Veiga, M. Merino, D. Fernandez and R. Lozano, J. Therm. Anal. Calorim., 2002, 68, 511-516.

45 P. Cloudy, J. Letoffe, P. Germain, J. Bastide, A. Bayol, S. Blasquez, R. Rao and B. Gonzalez, J. Therm. Anal. Calorim., 1991, 37, 2497-2506. 
46 O. Aleem, B. Kuchekar, Y. Pore and S. Late, J. Pharm. Biomed. Anal., 2008, 47, 535-540.

47 L. Liu and S. Zhu, J. Pharm. Biomed. Anal., 2006, 40, 122-127.

48 R. Bortolomeazzi, N. Sebastianutto, R. Toniolo and A. Pizzariello, Food Chem., 2007, 100, 1481-1489.
49 B. Shyamala, M. M. Naidu, G. Sulochanamma and P. Srinivas, J. Agric. Food Chem., 2007, 55, 77387743.

50 S. Santosh Kumar, K. Priyadarsini and K. Sainis, Redox Rep., 2002, 7, 35-40. 